\title{
COMPACTNESS OF THE NEUMANN-POINCARÉ OPERATOR
}

\author{
BY \\ E. J. SPECHT AND H. T. JONES( $\left.{ }^{(}\right)$
}

1. Introduction. The Neumann-Poincaré integral equation arises in connection with the Dirichlet and Neumann problems of potential theory and in connection with conformal mapping. Warschawski [6] has proved the compactness of the integral operator involved here under what seem to be natural smoothness conditions on the boundary curve, for the case where the boundary is a single contour. Because this proof relies heavily upon complex function theory, it does not extend easily to higher dimensions. It is the purpose of the present paper to give a proof, for the case of several contours, which will extend readily to higher dimensions.

2. Definitions. Let $\mathscr{B}_{1}, \ldots, \mathscr{B}_{m}$ be bounded nonintersecting contours in the plane whose interiors are disjoint, and let $\zeta_{j}$ be the standard representation $\left({ }^{2}\right)$ of $\mathscr{B}_{j}$. Let $s_{0}=0$, let $s_{j}$ be the sum of the lengths of $\mathscr{B}_{1}, \ldots, \mathscr{B}_{j}$, and let $\mathscr{I}=\left[0, s_{m}\right]$. Let $\zeta$ be the function defined on $\mathscr{I}$ so that $\zeta s=\zeta_{1} s$ for all $s$ in $\left[0, s_{1}\right]$ and $\zeta s=\zeta_{j}\left(s-s_{j-1}\right)$ for all $s$ in $\left(s_{j-1}, s_{j}\right], j=2, \ldots, m$, and let $\mathscr{B}$ be the range of $\zeta$.

Let $A$ be the function defined for all ordered pairs $(s, t)$ such that $\zeta s$ and $\zeta t$ both belong to $\mathscr{B}_{j}$ for some $j=1, \ldots, m$ as follows:

$$
\begin{aligned}
A(s, t) & =s-t & & \text { if }|s-t|<\frac{1}{2}\left(s_{j}-s_{j-1}\right) ; \\
& =(s-t)+\left(s_{j}-s_{j-1}\right) \operatorname{sgn}(t-s) & & \text { if }|s-t| \geqq \frac{1}{2}\left(s_{j}-s_{j-1}\right) .
\end{aligned}
$$

A function $\alpha$ defined on $\mathscr{I}$ will be said to satisfy a Hölder condition on $\mathscr{B}$ if and only if $|\alpha s-\alpha t| \leqq a|A(s, t)|^{b}$ for some numbers $a$ and $b$ such that $a>0$ and $0<b \leqq 1$. Continuity on $\mathscr{B}$ is defined analogously. Also, the derivative $D \alpha$ of $\alpha$ is that function whose value at $t$ is $\lim _{A(s, t) \rightarrow 0}(\alpha s-\alpha t) / A(s, t)$.

It is assumed that $\zeta$ has a derivative $D \zeta$ which satisfies a Hölder condition on $\mathscr{B}$. If $\zeta=\xi+i \eta$, then $D \xi$ and $D \eta$ satisfy the same Hölder condition on $\mathscr{B}$ as does $D \zeta$.

3. The space $\mathscr{P}$ and the operator $T$. Let $c$ be a number such that $c>|\zeta s-\zeta t|$ for all $s$ and $t$ in $\mathscr{I}$, and let the function $\Lambda$ be defined on $\mathscr{I} \times \mathscr{I}$ (except at points $(s, t)$ where $\left.\zeta_{s}=\zeta_{t}\right)$ by the equality $\Lambda(s, t)=\log \left(c /\left|\zeta_{s}-\zeta_{t}\right|\right)$. For any function $\alpha$ defined

Received by the editors July 8, 1968 .

(1) This research was supported by the National Science Foundation under grant NSF G-4142 and by the Office of Naval Research under contract Nonr-3911(00).

$\left({ }^{2}\right)$ A contour $\mathscr{B}$ is the range of a mapping $\alpha$ of a closed interval $[a, b]$ into the plane such that $\alpha$ is continuous and of bounded variation, $\alpha$ is one-to-one on $[a, b)$ and $\alpha a=\alpha b$. If $\sigma t$ denotes the total variation of $\alpha$ on the interval $[a, t]$ for every $t$ in $[a, b]$, and if $\sigma^{*}$ is the inverse function of $\sigma$, then the composition $\alpha \sigma^{*}$ is the standard representation of $\mathscr{B}$. 
on $\mathscr{I}$, let the function $M$ be defined by the equality $M(s, t)=(\alpha s)(\alpha t) \Lambda(s, t)$. Then, if the double integral $\iint_{\mathscr{S} \times \mathscr{S}} M$ exists and is finite, it will be denoted by $\|\alpha\|^{2}$.

In subsequent proofs, use will be made of the fact that if $\|\alpha\|^{2}$ exists, then so do the corresponding iterated integrals, and the three are equal.

THEOREM 3.1. If $\alpha$ is a continuous function on $\mathscr{I}$, then $\|\alpha\|^{2} \geqq 0$. Moreover, $\|\alpha\|^{2}=0$ if and only if $\alpha=0$.

A proof of this theorem for the case of a single contour with continuous curvature is given on pages $157-159$ of [1].

LEMMA 3.1. If $\|\alpha\|^{2}$ exists and is finite, then $\alpha$ is summable on $\mathscr{I}$.

Proof. If $\|\alpha\|^{2}$ exists, then $\int_{0}^{s_{m}} \alpha s \alpha t \Lambda(s, t) d s$ exists for almost all $t$ in $\mathscr{I}$, which implies that, for almost all $t$ in $\mathscr{I}$, the function $\alpha \cdot \Lambda(\iota, t)$, where $\iota$ is the identity function from $\mathscr{I}$ to $\mathscr{I}$, is summable on $\mathscr{I}$. Moreover, $1 / \Lambda(\iota, t)$ is continuous on $\mathscr{I}-\{t\}$ and bounded there. Therefore $\alpha$ is the product of a summable function and a bounded measurable function; hence it is summable on $\mathscr{I}$.

THEOREM 3.2. If $\|\alpha\|^{2}$ exists and is finite, then $\|\alpha\|^{2} \geqq 0$.

Proof. This theorem can be proved by making use of ideas in the proof of Lemma 1, p. 9, of [6].

It can be shown that, if $\|\alpha\|^{2}$ and $\|\beta\|^{2}$ exist and are finite, then the integral $\iint_{\sigma^{\prime}} P$, where $P(s, t)=(\alpha s)(\beta t) \Lambda(s, t)$, exists and is finite. This shows that the set of all functions $\alpha$ such that $\|\alpha\|^{2}$ exists and is finite can be regarded as an inner product space with the inner product $\langle\alpha, \beta\rangle$ given by the above integral. Let two functions $\alpha$ and $\beta$ of this space be called equivalent if and only if $\|\alpha-\beta\|=0$, and let a representative be chosen from each equivalence class. Let $\mathscr{P}$ be the inner product space of representatives.

To discuss the classical Neumann-Poincare integral equation, it is convenient to introduce the linear operator $\boldsymbol{T}$ in $\mathscr{P}$ defined as follows. Let $\Gamma$ be the function defined for each pair $(x, y)$ of real numbers such that $x^{2}+y^{2} \neq 0$ by the equality

$$
\Gamma(x, y)=\log \left(c / \sqrt{ }\left(x^{2}+y^{2}\right)\right),
$$

let $D_{u_{t}} \Gamma$ be its directional derivative in the direction of the vector $u_{t}=-D \eta t$ $+i D \xi t$, and let

$$
K(s, t)=(1 / \pi) D_{u_{t}} \Gamma(\xi s-\xi t, \eta s-\eta t),
$$

for each ordered pair $(s, t)$ in $\mathscr{I} \times \mathscr{I}$ except $\left(s_{1}, 0\right),\left(0, s_{1}\right)$, and those for which $s=t$.

Since $K$ is continuous on $\mathscr{I} \times \mathscr{I}$ except on a set of measure zero, it is measurable there. Moreover (see $\$ 6$, Property (iii)), because the function whose value at $s$ is $\int_{\mathscr{S}}|K(s, \iota)|$ satisfies a Hölder condition on $\mathscr{B}$, it is bounded and measurable on $\mathscr{I}$. If $\|\alpha\|^{2}$ exists, then, by Lemma 3.1, $\alpha$ is summable on $\mathscr{I}$, and it follows that the integral $\int_{0}^{s_{m}} \int_{0}^{s_{m}}|\alpha s K(s, t)| d t d s$ exists. Then, by Fubini's theorem, the function $T \alpha$ whose value at $t$ is $\int_{\mathscr{S}}(\alpha \cdot K(\iota, t))$, is summable on $\mathscr{I}$. 
THEOREM 3.3. If $\|\alpha\|^{2}$ exists, then $\|T \alpha\|^{2}$ exists.

Proof. It is not difficult to show that the function $\bar{H}$ such that

$$
\bar{H}(s, t)=\int_{\sigma}(|K(s, \iota)| \cdot \Lambda(\iota, t))
$$

is continuous on $\mathscr{I} \times \mathscr{I}$ (see $\S 6$, Property (v)) and then that, for every function $\alpha$ for which $\|\alpha\|^{2}$ exists, the function $\beta$ such that $\beta x=\int_{\mathscr{S}}(|\alpha| \cdot \bar{H}(\iota, x))$ is continuous on $\mathscr{I}$. If $\|\alpha\|^{2}$ exists, $T \alpha$ is summable on $\mathscr{I}$, and hence $\int_{\mathscr{I}}(\beta \cdot|\boldsymbol{T} \alpha|)$ exists. But

$$
\int_{\mathcal{S}}(\beta \cdot|\boldsymbol{T} \alpha|)=\int_{0}^{s_{m}} \int_{0}^{s_{m}} \int_{0}^{s_{m}}|\alpha x||K(x, t)||\boldsymbol{T} \alpha s| \Lambda(t, s) d t d x d s=\|\boldsymbol{T} \alpha\|^{2},
$$

by virtue of a generalization of Fubini's theorem to three-place functions. This completes the proof.

Therefore $T$ is an operator in $\mathscr{P}$. For $n=2,3,4, \ldots, T^{n} \alpha t=\int_{\mathscr{S}}\left(\alpha \cdot K_{n}(\iota, t)\right)$, where $K_{n}(s, t)=\int_{s}\left(K_{n-1}(s, \imath) \cdot K(\iota, t)\right)$ and $K_{1}=K$. Since the function $H$ for which $H(s, t)=\int_{s}(K(s, \imath) \cdot \Lambda(\iota, t))$ has the property that $H(s, t)=H(t, s)$, as can be shown from Green's second identity, it follows that, for every $\alpha$ and $\beta$ in $\mathscr{P},\langle T \alpha, \beta\rangle$ $=\langle\alpha, T \beta\rangle$.

4. Definition and properties of $\Omega_{n}$. In classical potential theory (see, for example, [5, p. 299]), it is shown that there exists an orthonormal set $\left\{\varphi_{1}, \ldots, \varphi_{m}\right\}$ of functions such that $\boldsymbol{T} \varphi_{j}=\varphi_{j}$ for $j=1, \ldots, m$. Moreover, these functions have the properties that, for some nonzero real numbers $c_{1}, \ldots, c_{m}$,

$$
\begin{aligned}
& \int_{s_{i}-1}^{s_{j}} \varphi_{k}=c_{k} \quad \text { if } k=j \\
& =0 \text { if } k \neq j,
\end{aligned}
$$

for $k, j=1, \ldots, m$, and

$$
\begin{aligned}
\int_{\mathscr{G}}\left(\varphi_{j} \cdot \Lambda(s, \iota)\right) & =1 / c_{j} & & \text { if } \zeta s \in \mathscr{B}_{j} \\
& =0 & & \text { if } \zeta_{s \in \mathscr{B}_{k}} \text { for } k \neq j,
\end{aligned}
$$

for $j=1, \ldots, m$. Since $\varphi_{j}=T^{n} \varphi_{j}$ for $j=1, \ldots, m$ and for every positive integer $n$, it follows from Property (iv) (see §6) that the $\varphi$ 's satisfy a Hölder condition on $\mathscr{B}$.

THEOREM 4.1. If $n$ is a sufficiently large positive integer, there exists a function $\Omega_{n}$ such that, for each $s$ and $t$ in $\mathscr{I}, K_{n}(s, t)=\int_{\mathscr{S}}\left(\Omega_{n}(\imath, t) \cdot \Lambda(\iota, s)\right)$, and, for each $t$ in $\mathscr{I}$, $\Omega_{n}(\iota, t)$ is continuous on $\mathscr{B}$.

For a proof of this theorem, see [6, p. 15].

Let $I$ be the identity mapping of the complex plane onto itself, and, for each $t$ in $\mathscr{I}$, let $\Phi_{e}^{\prime}(I, t)$ be the solution of the exterior Dirichlet problem for $\mathscr{B}$ with boundary values $K_{n}(\iota, t)$. Since, for each $t$ in $\mathscr{I}, D_{1} K_{n}(\iota, t)$ satisfies a Hölder 
condition on $\mathscr{B}$ if $n$ is sufficiently large (see $\S 6$, Property (vii)), it follows (see [3, p. 111]) that for each $(s, t)$ in $\mathscr{I} \times \mathscr{I}, \lim _{z \rightarrow \zeta s} D_{u_{s}} \Phi_{e}^{\prime}(z, t)$ exists, and the function whose value at $s$ is given by this limit is continuous on $\mathscr{B}$. Therefore, since the operator $\boldsymbol{T}$ is compact in $\mathscr{L}_{2}\left[0, s_{m}\right]$ (see [7, pp. 326-329]), it follows by the Fredholm theory that there is a function $\bar{\Omega}_{n}$ such that $\bar{\Omega}_{n}(\iota, t)$ is continuous on $\mathscr{B}$ and such that

$$
\lim _{z \rightarrow \infty} \bar{\Phi}_{e}(z, t)=0
$$

and

$$
\lim _{z \rightarrow \zeta s} D_{u_{s}} \bar{\Phi}_{e}(z, t)=\lim _{z \rightarrow \zeta s} \Phi_{e}^{\prime}(z, t)
$$

where

$$
\bar{\Phi}_{e}(z, t)=\int_{\mathscr{I}}\left(\bar{\Omega}_{n}(\iota, t) \cdot \log (c /|z-\zeta|)\right)
$$

for all $t$ in $\mathscr{I}$ and all $z$ in $\mathscr{B}_{e}$, the unbounded region determined by $\mathscr{B}$. Since $\Phi_{e}^{\prime}(I, t)$ $-\bar{\Phi}_{e}(I, t)$ is a function which is harmonic in $\mathscr{B}_{e}$ and whose normal derivative is zero on $\mathscr{B}$, it follows by the uniqueness, to within an additive constant, of the solution of the Neumann problem, that there is a function $\gamma$ on $\mathscr{I}$ to $\mathscr{I}$ such that

$$
\Phi_{e}^{\prime}(z, t)-\bar{\Phi}_{e}(z, t)=\gamma t
$$

for all $z$ in $\mathscr{B}_{e}$. An explicit expression for the function $\Omega_{n}$ whose existence is asserted in Theorem 4.1 is given by the equality

$$
\Omega_{n}(\iota, t)=\bar{\Omega}_{n}(\iota, t)+(\gamma t) \omega,
$$

where $\omega=\sum_{j=1}^{m} c_{j} \varphi_{j}$.

Let

$$
\Phi_{i}(z, t)=\int_{\mathscr{S}}\left(\Omega_{n}(\iota, t) \cdot \log (c /|z-\zeta|)\right)
$$

for each $z$ in $\mathscr{B}_{i}$, where $\mathscr{B}_{i}$ is the union of the interior regions determined by $\mathscr{B}$, let

$$
\Psi_{i}(s, t)=\lim _{z \rightarrow \zeta s} D_{u_{s}} \Phi_{i}(z, t),
$$

let

$$
\Phi_{e}(z, t)=\int_{\zeta}\left(\Omega_{n}(\iota, t) \cdot \log (c /|z-\zeta|)\right)
$$

for each $z$ in $\mathscr{B}_{e}$, and let

$$
\Psi_{e}(s, t)=\lim _{z \rightarrow \zeta s} D_{u_{s}} \Phi_{e}(z, t) .
$$

Then, by the well-known behavior of the normal derivative of the potential due to a single-layer distribution,

$$
\Omega_{n}=(1 / 2 \pi)\left(\Psi_{e}-\Psi_{i}\right)
$$


LEMMA 4.1. There exist functions $\Delta_{i}$ and $\Delta_{e}$ such that, for all $t$ in $\mathscr{I}$,

$$
\frac{1}{\pi} \int_{0}^{s_{m}} \Delta_{i}(s, t) D_{u_{s}} \Gamma(x-\xi s, y-\eta s) d s=\Phi_{i}(z, t)
$$

for all $z$ in $\mathscr{B}_{i}$, where $z=x+i y$; and

$$
\begin{aligned}
\frac{1}{\pi} \int_{0}^{s_{m}} \Delta_{e}(s, t) D_{u_{s}} \Gamma(x-\xi s, y-\eta s) d s \\
\quad+\int_{0}^{s_{m}}\left(\sum_{j=1}^{m}\left(\varphi_{j} s\right)\left(\varphi_{j} t\right)-(\omega s)(\gamma t)\right) \log \frac{c}{|\zeta s-z|} d s+\gamma t=\Phi_{e}^{\prime}(z, t)
\end{aligned}
$$

for all $z$ in $\mathscr{B}_{e}$. Moreover, for each $s$ in $\mathscr{I}, D_{1} \Delta_{i}(s, \iota), D_{1} \Delta_{e}(s, \iota), D_{1} \Delta_{i}(\iota, s)$, and $D_{1} \Delta_{e}(\iota, s)$ exist and satisfy Hölder conditions on $\mathscr{B}$, uniformly with respect to $s$.

Proof. Since $\lim _{z \rightarrow \zeta v} \Phi_{i}(z, t)=K_{n}(v, t)$, one can show, by using the well-known discontinuous behavior of a potential arising from a double-layer distribution and the uniqueness of the solution of the Dirichlet problem, that there exists a function $\Delta_{i}$ satisfying equation (4) if and only if the integral equation

$$
\Delta_{i}(v, t)+\int_{\mathscr{I}}\left(\Delta_{i}(\iota, t) \cdot K(v, \iota)\right)=K_{n}(v, t)
$$

has a solution. Since (see [7, pp. 326-329]) the operator $\boldsymbol{T}$ is compact in $\mathscr{L}_{2}\left[0, s_{m}\right]$, the Fredholm theory is applicable. The homogeneous equation corresponding to (6) has no nontrivial solutions, and hence for each $t$ in $\mathscr{I}$, equation (6) has a unique solution $\Delta_{i}(\iota, t)$. It satisfies the equation

$$
\Delta_{i}(v, t)+(-1)^{p+1} \int_{\mathscr{J}}\left(\Delta_{i}(\iota, t) \cdot K_{p}(v, \iota)\right)=\sum_{j=0}^{p-1}(-1)^{j} K_{n+j}(v, t)
$$

for each positive integer $p$, as may be proved by induction on $p$, equation (6) being the statement for $p=1$. A solution of this equation for the case where $p=2 n$ is given by the expression

$$
\Delta_{i}(v, t)=\sum_{j=0}^{2 n-1}(-1)^{j}\left[K_{n+j}(v, t)+\int_{\mathscr{I}}\left(K_{n+j}(\iota, t) \cdot P(v, \iota)\right)\right],
$$

where $P$ is the Fredholm resolvent for the kernel $K_{2 n}$. From this expression it follows that $\Delta_{i}$ is bounded on $\mathscr{I} \times \mathscr{I}$ and $\Delta_{i}(v, \imath)$ satisfies a Hölder condition on $\mathscr{B}$ uniformly in $v$, since the functions $\sum_{j=0}^{2 n-1}(-1)^{j} K_{n+j}$ and $P$ are both bounded on $\mathscr{I} \times \mathscr{I}$, and the function $\sum_{j=0}^{2 n-1}(-1)^{j} K_{n+j}(v, \imath)$ satisfies a Hölder condition on $\mathscr{B}$ uniformly in $v$ (see $\S 6$, Property (iv)). Moreover, if $p$ is taken to be $2 n$ in equation (7), it follows that, for each $v$ in $\mathscr{I}, D_{1} \Delta_{i}(v, \iota)$ exists and

$$
D_{1} \Delta_{i}(v, t)=\int_{\mathscr{I}}\left(\Delta_{i}(\iota, t) \cdot D_{1} K_{2 n}(v, \iota)\right)+\sum_{j=0}^{2 n-1}(-1)^{j} D_{1} K_{n+j}(v, t),
$$

differentiation under the integral sign being justified because $\Delta^{i}(\iota, t) \cdot D_{1} K_{2 n}(v, \iota)$ is 
summable for all $v$ and $t$ in $\mathscr{I}$. Now from the facts that $D_{1} K_{2 n}$ is bounded on $\mathscr{I} \times \mathscr{I}$ and that $D_{1} K_{n}(v, \imath), \ldots, D_{1} K_{3 n-1}(v, \imath)$, and $\Delta_{i}(v, \imath)$ satisfy Hölder conditions on $\mathscr{B}$ uniformly with respect to $v$, it follows by (8) that $D_{1} \Delta_{i}(v, \iota)$ satisfies a Hölder condition on $\mathscr{B}$, uniformly with respect to $v$.

Furthermore, since $\Delta_{i}$ is bounded on $\mathscr{I} \times \mathscr{I}$ and $D_{1} K_{n}(\iota, t), \ldots, D_{1} K_{3 n-1}(\iota, t)$ satisfy Hölder conditions on $\mathscr{B}$ uniformly with respect to $t$ (see $\S 6$, Property (iii)), it follows from (8) that $D_{1} \Delta_{i}(\iota, t)$ satisfies a Hölder condition on $\mathscr{B}$, uniformly with respect to $t$. Similarly, $D_{1} \Delta_{i}$ is bounded on $\mathscr{I} \times \mathscr{I}$. This completes the proof of the statements about $\Delta_{i}$ in the lemma.

A similar treatment of $\Phi_{e}^{\prime}$ is impossible because the integral equation corresponding to (6) in this case would be

$$
\Delta_{e}(v, t)-\int_{\mathcal{G}}\left(\Delta_{e}(\iota, t) \cdot K(v, \iota)\right)=-K_{n}(v, t),
$$

which has no solution because the associated homogeneous equation

$$
\Delta_{e}(v, t)-\int_{\mathcal{S}}\left(\Delta_{e}(\iota, t) \cdot K(\iota, v)\right)=0
$$

has solutions which are not orthogonal in $\mathscr{L}_{2}\left[0, s_{m}\right]$ to the function $-K_{n}(\iota, t)$. However, the integral equation

$$
\Delta_{e}(v, t)-\int_{\mathcal{G}}\left(\Delta_{e}(\iota, t) \cdot K(v, \imath)\right)=-K_{n}(v, t)+\sum_{j=1}^{m}\left(\mu_{j} v\right)\left(\varphi_{j} t\right),
$$

where $\mu_{j} v=\int_{\mathcal{G}}\left(\varphi_{j} \cdot \Lambda(v, \iota)\right)$, does have a solution $\Delta_{e}(\iota, t)$, which then has the properties that

$$
\lim _{z \rightarrow \zeta v} \Xi(z, t)=K_{n}(v, t)-\sum_{j=1}^{m}\left(\mu_{j} v\right)\left(\varphi_{j} t\right)
$$

and $\lim _{z \rightarrow \infty} \Xi(z, t)=0$, where

$$
\Xi(z, t)=(1 / \pi) \int_{0}^{s_{m}} \Delta_{e}(s, t) D_{u_{s}} \Gamma(x-\xi s, y-\eta s) d s,
$$

$z=x+i y$. Hence the function whose value at $z$ is

$$
\Xi(z, t)+\sum_{j=1}^{m}\left(\varphi_{j} t\right) \int_{\delta}\left(\varphi_{j} \cdot \log (c /|\zeta-z|)\right)
$$

has the same limit on $\mathscr{B}$ as does $\Phi_{e}^{\prime}(I, t)$, but its limit at infinity is zero, whereas $\lim _{z \rightarrow \infty} \Phi_{e}^{\prime}(z, t)=\gamma t$. From these considerations, equation (5) follows.

As can be proved by induction, $\Delta_{e}(\iota, t)$ also satisfies the equation

$$
\Delta_{e}(v, t)-\int_{\mathcal{f}}\left(\Delta_{e}(\iota, t) \cdot K_{p}(v, \iota)\right)=-\sum_{k=0}^{p-1} K_{n+k}(v, t)+p \sum_{j=1}^{m}\left(\mu_{j} v\right)\left(\varphi_{j} t\right),
$$


for each positive integer $p$. A solution of this equation for the case where $p=2 n$ is given by the relation

$$
\begin{aligned}
\Delta_{e}(v, t)= & -\sum_{k=0}^{2 n-1} K_{n+k}(v, t)+2 n \sum_{j=1}^{m}\left(\mu_{j} v\right)\left(\varphi_{j} t\right) \\
& +\int_{\mathcal{G}}\left(\left(\sum_{k=0}^{2 n-1} K_{n+k}(\iota, t)+2 n \sum_{j=1}^{m}\left(\varphi_{j} t\right) \mu_{j}\right) \cdot P(v, \iota)\right) .
\end{aligned}
$$

The argument to show that $D_{1} \Delta_{e}$ has the properties stated in the lemma now proceeds like that for $D_{1} \Delta_{i}$.

LEMMA 4.2. For each $s$ in $\mathscr{I}, \Omega_{n}(s, \iota)$ is continuous on $\mathscr{B}$, and the continuity is uniform with respect to $s$.

Proof. For each $(s, t)$ in $\mathscr{I} \times \mathscr{I}$,

and

$$
\Psi_{i}(s, t)=\int_{\mathscr{\zeta}}\left(D_{1} \Delta_{i}(\iota, t) \cdot E(\iota, s)\right)
$$

where

$$
\Psi_{e}(s, t)=\int_{\mathcal{\sigma}}\left(D_{1} \Delta_{e}(\iota, t) \cdot E(\iota, s)\right)+\sum_{j=1}^{m}\left(\varphi_{j} s\right)\left(\varphi_{j} t\right),
$$

$$
E(v, s)=(1 / 2 \pi)[(\xi s-\xi v)(D \xi s)+(\eta s-\eta v)(D \eta s)] /\left[(\xi s-\xi v)^{2}+(\eta s-\eta v)^{2}\right],
$$

and where the Cauchy principal value of each integral is understood (see [2, p. 46]). Now if $r$ is a sufficiently small positive number, there exist functions $\theta_{1}$ and $\theta_{2}$, defined on the set

such that

$$
\{v: 0<|A(v, s)| \leqq r\}
$$

and

where

$$
\left|\theta_{1} v-s\right|<|A(v, s)|, \quad\left|\theta_{2} v-s\right|<|A(v, s)|
$$

$$
E(v, s)=Z(v, s) / A(s, v)
$$

$$
Z(v, s)=\left[\left(D \xi \theta_{1} v\right)(D \xi s)+\left(D \eta \theta_{2} v\right)(D \eta s)\right] /\left[\left(D \xi \theta_{1} v\right)^{2}+\left(D \eta \theta_{2} v\right)^{2}\right] .
$$

Also, for each sufficiently small positive number $r$, there is a positive number $\kappa r$ such that $|E(w, s)|<\kappa r$ for all $(w, s)$ in the set

$$
\mathscr{I} \times \mathscr{I}-\{(w, s):|A(w, s)| \leqq r\} .
$$

For each $s$ in $\mathscr{I}$, let $\beta_{s}$ be the inverse of the function $A(s, \imath)$, and let $v_{1}=\beta_{s}(-r)$ and $v_{2}=\beta_{s} r$. Then, by Lemma 4.1 , there exist numbers $f_{1}$ and $g_{1}$ such that $f_{1}>0$, $0<g_{1} \leqq 1$, and for all $h$ for which $(t+h) \in \mathscr{I}$,

$$
\begin{aligned}
\left|\Psi_{i}(s, t+h)-\Psi_{i}(s, t)\right| \leqq & 2(\kappa r) f_{1} s_{m}|A(t+h, t)|^{g_{1}} \\
& +\left|(c p v) \int_{v_{1}}^{v_{2}}\left[\left(D_{1} \Delta_{i}(\iota, t+h)-D_{1} \Delta_{i}(\iota, t)\right) \cdot E(\iota, s)\right]\right|
\end{aligned}
$$


Since $D \xi, D \eta$, and $D_{1} \Delta_{i}(\iota, t)$ satisfy Hölder conditions on $\mathscr{B}$ and since $(D \xi)^{2}$ $+(D \eta)^{2}=1$, it can be shown by standard arguments (see, for example, $\left[4, \S 6,2^{\circ}\right.$ and $3^{\circ}$ ) ) that, for $r$ sufficiently small, for some positive number $d_{1}$ and some $d_{2}$ such that $0<d_{2} \leqq 1$, for all $s$ in $\mathscr{I}$, and for all $u$ such that $0<u \leqq r$,

$$
\left|D_{1} \Delta_{i}\left(\beta_{s}(-u), t\right) Z\left(\beta_{s}(-u), s\right)-D_{1} \Delta_{i}\left(\beta_{s} u, t\right) Z\left(\beta_{s} u, s\right)\right| \leqq d_{1}|2 u|^{d_{2}},
$$

whence

$$
\begin{aligned}
\mid(c p v) & \int_{v_{1}}^{v_{2}}\left(D_{1} \Delta_{i}(\iota, t) \cdot E(\iota, s)\right) \mid \\
& =\lim _{q \rightarrow 0+}\left|\int_{v_{1}}^{\beta_{s}(-q)}\left(D_{1} \Delta_{i}(\iota, t) \cdot E(\iota, s)\right)+\int_{\beta_{s} q}^{v_{2}}\left(D_{1} \Delta_{i}(\iota, t) \cdot E(\iota, s)\right)\right| \\
& =\lim _{q \rightarrow 0+}\left|\int_{q}^{r}\left[D_{1} \Delta_{i}\left(\beta_{s}(-u), t\right) Z\left(\beta_{s}(-u), s\right)-D_{1} \Delta_{i}\left(\beta_{s} u, t\right) Z\left(\beta_{s} u, s\right)\right] \frac{1}{u} d u\right| \\
& \leqq \lim _{q \rightarrow 0+} \frac{2^{d_{2}} d_{1}\left(r^{d_{2}}-q^{d_{2}}\right)}{d_{2}}=d_{1}(2 r)^{d_{2}} / d_{2} .
\end{aligned}
$$

The integral

$$
\left|(c p v) \int_{v_{1}}^{v_{2}}\left(D_{1} \Delta_{i}(\iota, t+h) \cdot E(\iota, s)\right)\right|
$$

can be treated in exactly the same way. Therefore

$$
\left|\Psi_{i}(s, t+h)-\Psi_{i}(s, t)\right| \leqq 2(\kappa r) f_{1} s_{m}|h|^{g_{1}}+2 d_{1}(2 r)^{d_{2}} / d_{2} .
$$

By choosing $r$ sufficiently small, and then $h$, it is possible to make

$$
\left|\Psi_{i}(s, t+h)-\Psi_{i}(s, t)\right|
$$

arbitrarily small, and the choice of $h$ is independent of $s$. This proves that $\Psi_{i}(s, \iota)$ is continuous and that the continuity is uniform with respect to $s$. The proof that $\Psi_{e}(s, \iota)$ has the same property is similar. The lemma then follows from equation (3).

LEMMA 4.3. The function whose value at $t$ is $\int_{\mathscr{G}}\left|\Omega_{n}(\iota, t)\right|$ is continuous and bounded on $\mathscr{I}$.

Proof. This follows from the uniform continuity in Lemma 4.2.

Lemma 4.4. For some positive number $a_{0},\left\|\Omega_{n}(\iota, t)\right\| \leqq a_{0}$ for all $t$ in $\mathscr{I}$. For some numbers $f$ and $g$ such that $f>0$ and $0<g \leqq 1$, for all $t$ and $t+h$ in $\mathscr{I}$,

$$
\left\|\Omega_{n}(\iota, t+h)-\Omega_{n}(\iota, t)\right\| \leqq f|A(t+h, t)|^{g} .
$$

Proof. The first inequality follows from Lemma 4.3 and the inequality

$$
\begin{aligned}
\left\|\Omega_{n}(\iota, t)\right\|^{2} & =\int_{0}^{s_{m}} \int_{0}^{s_{m}} \Omega_{n}(u, t) \Omega_{n}(v, t) \Lambda(u, v) d u d v \\
& =\int_{0}^{s_{m}} \Omega_{n}(u, t) K_{n}(u, t) d u \leqq k_{n} \int_{0}^{s_{m}}\left|\Omega_{n}(u, t)\right| d u,
\end{aligned}
$$

where $k_{n}$ is a number such that $\left|K_{n}(u, t)\right| \leqq k_{n}$ (see $\S 6$, Property (i)). 
The second part of the lemma follows from Lemma 4.2 and the fact that

$$
\begin{aligned}
\left\|\Omega_{n}(\iota, t+h)-\Omega_{n}(\iota, t)\right\|^{2} & \left.=\int_{\mathscr{I}}\left[\Omega_{n}(\iota, t+h)-\Omega_{n}(\iota, t)\right) \cdot\left(K_{n}(\iota, t+h)-K_{n}(\iota, t)\right)\right] \\
& \leqq b_{0}|A(t+h, t)|^{g} \int_{\mathscr{I}}\left|\Omega_{n}(\iota, t+h)-\Omega_{n}(\iota, t)\right|,
\end{aligned}
$$

where the fact that $K_{n}(s, \iota)$ satisfies a Hölder condition on $\mathscr{B}$ has been exploited.

\section{Properties of $T$.}

LEMMA 5.1. The operator $T$ is bounded on $\mathscr{P}$.

Proof. For $n$ sufficiently large, for each $\alpha$ in $\mathscr{P}$ and each $t$ in $\mathscr{I}$,

$$
\left|T^{n} \alpha t\right|=\left|\left\langle\Omega_{n}(\iota, t), \alpha\right\rangle\right| \leqq\left\|\Omega_{n}(\iota, t)\right\| \cdot\|\alpha\| \leqq a_{0}\|\alpha\|
$$

by Lemma 4.4. Therefore

$$
\left\|\boldsymbol{T}^{n} \alpha\right\|^{2} \leqq \int_{0}^{s_{m}} \int_{0}^{s_{m}}\left|\boldsymbol{T}^{n} \alpha S\right|\left|\boldsymbol{T}^{n} \alpha t\right| \Lambda(s, t) d s d t \leqq a_{0}^{2}\|\alpha\|^{2} \int_{0}^{s_{m}} \int_{0}^{s_{m}} \Lambda(s, t) d s d t,
$$

which shows that $\boldsymbol{T}^{n}$ is bounded in $\mathscr{P}$ for all sufficiently large $n$. In particular, $\boldsymbol{T}^{2^{p}}$ is bounded for sufficiently large $p$, from which it follows that $\boldsymbol{T}$ is bounded on $\mathscr{P}$ by virtue of the fact that

$$
\left\|T^{2^{p-1}} \alpha\right\|^{2}=\left\langle T^{\iota^{p-1}} \alpha, T^{2^{p-1}} \alpha\right\rangle=\left\langle T^{2^{p}} \alpha, \alpha\right\rangle \leqq\left\|T^{2^{p}} \alpha\right\| \cdot\|\alpha\|
$$

for all $\alpha$ in $\mathscr{P}$.

Now let $\mathscr{H}$ be the Hilbert space obtained by completing the inner product space $\mathscr{P}$, and let the extension of $\boldsymbol{T}$ to $\mathscr{H}$ by continuity be denoted by the same symbol.

THEOREM 5.1. The operator $T$ is compact in $\mathscr{H}$.

Proof. Let $\mathscr{F}$ be any bounded set in $\mathscr{H}$, so that, for some positive number $c_{0}$, $\|\alpha\| \leqq c_{0}$ for all $\alpha$ in $\mathscr{F}$. Let $\mathscr{G}=\boldsymbol{T}^{2 n} \mathscr{F}$, where $n$ is the integer introduced in Theorem 4.1. Then every element of $\mathscr{G}$ is a function continuous on $\mathscr{B}$. To see this, let $\alpha$ be any element of $\mathscr{F}$. Then there exists a sequence of functions $\beta_{1}, \beta_{2}, \beta_{3}, \ldots$ in $\mathscr{P}$ such that $\lim _{k \rightarrow \infty} \beta_{k}=\alpha$. Moreover, $T^{n} \alpha=\lim _{k \rightarrow \infty} T^{n} \beta_{k}$. Because this sequence converges, there exists a $\bar{c}_{0}$ such that $\left\|\beta_{k}\right\| \leqq \bar{c}_{0}$ for $k=1,2,3, \ldots$, and hence, for all $t$ in $\mathscr{I}$, $\left|T^{n} \beta_{k} t\right| \leqq a_{0} \bar{c}_{0}$, by virtue of the relation (10). Then, for all $s$ and $s+h$ in $\mathscr{I}$,

$$
\begin{aligned}
\left|\boldsymbol{T}^{2 n} \beta_{k}(s+h)-\boldsymbol{T}^{2 n} \beta_{k} s\right| & =\left|\int_{\mathcal{S}}\left[\left(K_{n}(\iota, s+h)-K_{n}(\iota, s)\right) \cdot\left(\boldsymbol{T}^{n} \beta_{k}\right)\right]\right| \\
& \leqq a_{0} b_{0} \bar{c}_{0}|A(s+h, s)|^{g},
\end{aligned}
$$

where the same Hölder condition as was exploited in (9) has been used here. From the fact that

$$
\left|\boldsymbol{T}^{2 n} \beta_{k} t-\boldsymbol{T}^{2 n} \beta_{j} t\right| \leqq\left\|\Omega_{2 n}(\iota, t)\right\| \cdot\left\|\beta_{k}-\beta_{j}\right\| \leqq a_{0}\left\|\beta_{k}-\beta_{j}\right\|,
$$


it follows that the sequence of continuous functions $T^{2 n} \beta_{1}, T^{2 n} \beta_{2}, T^{2 n} \beta_{3}, \ldots$ is uniformly convergent, so that the limit function is continuous on $\mathscr{B}$. Since this pointwise convergence implies convergence in the norm, the limit function is $T^{2 n} \alpha$.

Now the functions of $\mathscr{G}$ are uniformly bounded because, by the relation (10), $\left|T^{2 n} \alpha t\right| \leqq a_{0}^{\prime} c_{0}$, for some positive number $a_{0}^{\prime}$. Moreover, $\mathscr{G}$ is equicontinuous because, for each $\alpha$ in $\mathscr{F}$, and each $t$ and $t+h$ in $\mathscr{I}$,

$$
\left|T^{2 n} \alpha(t+h)-T^{2 n} \alpha t\right| \leqq\left\|\Omega_{2 n}(\iota, t+h)-\Omega_{2 n}(\iota, t)\right\| \cdot\|\alpha\| \leqq c_{0} f|A(t+h, t)|^{g}
$$

by Lemma 4.4. By Ascoli's theorem, every sequence of functions in $\mathscr{G}$ contains a pointwise convergent subsequence, which subsequence also converges in the norm. This shows that $\boldsymbol{T}^{2 n}$ is compact in $\mathscr{H}$. Since $\boldsymbol{T}$ is self-adjoint, it follows (see, for example, [7, p. 317], that $T$ is compact in $\mathscr{H}$.

From the first part of this proof, it follows that every characteristic vector of $\boldsymbol{T}$ is a function which satisfies a Hölder condition on $\mathscr{B}$ because every characteristic vector of $T$ is also a characteristic vector of $T^{2 n}$.

6. Properties of $K$. For convenience, certain properties of $K$ and its iterates are listed here. The set $\mathscr{S}$ is the set obtained by removing the points $\left(0, s_{1}\right),\left(s_{1}, 0\right)$, and all points of the diagonal (i.e., points of the form $(s, s)$ ) from the set $\mathscr{I} \times \mathscr{I}$. The number $b$ is the exponent of the Hölder condition satisfied by $D \zeta$.

(i) For each positive integer $n$, if $1-n b>0$, then $K_{n} \cdot|A|^{1-n b}$ is bounded on $\mathscr{P}$; if $1-n b<0$, then $K_{n}$ is bounded on $\mathscr{S}$. Hence, for sufficiently large $n$, it is possible to define $K_{n}$ at all points of $\mathscr{I} \times \mathscr{I}$ by continuity.

(ii) For each positive integer $n, D_{1} K_{n}$ exists and, if

$1-(n-1) b>0$, then $\left(D_{1} K_{n}\right) \cdot|A|^{2-n b}$ is bounded on $\mathscr{S}$; if

$1-(n-1) b<0$, then $\left(D_{1} K_{n}\right) \cdot|A|^{2-b}$ is bounded on $\mathscr{S}$.

(iii) If $\alpha$ is any function which is bounded and measurable on $\mathscr{I}$, then the function whose value at $t$ is $\int_{\mathscr{S}}\left(K_{n}(t, \imath) \cdot \alpha\right)$ satisfies a Hölder condition on $\mathscr{B}$ with exponent $\min \{1, n b\}$, for each positive integer $n$. In particular, if $q$ is a positive integer such that $K_{q}$ is bounded on $\mathscr{S}$, then $K_{q+n}(\iota, s)$ satisfies a Hölder condition on $\mathscr{B}$ with exponent $\min \{1, n b\}$ for all $s$ in $\mathscr{I}$.

(iv) If $\alpha$ is any function which is bounded and measurable on $\mathscr{I}$, then the function whose value at $s$ is $\int_{\mathscr{f}}(K(\iota, s) \cdot \alpha)$ satisfies a Hölder condition on $\mathscr{B}$ with exponent $b^{\prime}$, where $b^{\prime}$ is any number such that $0<b^{\prime}<b$. In particular, if $q$ is a positive integer such that $K_{q}$ is bounded on $\mathscr{S}$, then $K_{q+n}(s, \imath)$ satisfies a Hölder condition on $\mathscr{B}$ with exponent $b^{\prime}$ for every positive integer $n$ and for every $s$ in $\mathscr{I}$.

(v) There exist $a_{1}, a_{2}, b_{1}$, and $b_{2}$ such that $a_{1}>0, a_{2}>0,0<b_{1}<1,0<b_{2}<1$, and

$$
|H(s+h, t+k)-H(s, t)| \leqq a_{1}|A(s+h, s)|^{b_{1}}+a_{2}|A(t+k, t)|^{b_{2}}
$$

for all $(s, t)$ in $\mathscr{I} \times \mathscr{I}$ and all $h$ and $k$ sufficiently close to zero. This statement is also true if $H$ is replaced by $\bar{H}$. 
(vi) For each positive integer $n, D_{1} K_{n}(s, t)=(-1)^{n+1} D_{1} K_{n}(t, s)$ for all points $(s, t)$ of $\mathscr{S}$.

(vii) If $n$ is a sufficiently large positive integer, then $D_{1} K_{n}(\iota, s)$ and $D_{1} K_{n}(s, \iota)$ each satisfy a Hölder condition on $\mathscr{B}$ with exponent $b^{\prime}$, where $b^{\prime}$ is any number such that $0<b^{\prime}<b$, for all $s$ in $\mathscr{I}$.

Properties (i) and (ii) can be proved by induction. The proofs for the case $n=1$ follow from the equality

$$
K(s, t)=\frac{1}{\pi} \frac{(\eta s-\eta t) D \xi t-(\xi s-\xi t) D \eta t}{(\xi s-\xi t)^{2}+(\eta s-\eta t)^{2}}
$$

The induction argument for Property (ii) outlined by Warschawski (see [6, p. 12]) for the case $m=1$ extends easily to the case of several contours. Properties (i) and (ii) can then be used to prove (iii).

Property (iv) can be proved as follows. Suppose that $|\alpha s|<c_{0}$ for all $s$ in $\mathscr{I}$ and that $\zeta t$ and $\zeta(t+k)$ both belong to $\mathscr{B}_{j}$, and let $r$ be a positive number such that $r<\frac{1}{2}\left(s_{j}-s_{j-1}\right)$. Let $k$ be any number such that $2|k|<r$, and let

$$
\mathscr{E}_{1}=\{s: 2|k| \leqq|A(s, t)| \leqq r\}, \quad \mathscr{E}_{2}=\{s:|A(s, t)| \leqq 2|k|\} .
$$

Then

$$
\begin{aligned}
& \left|\int_{\mathscr{S}}(K(\iota, t+k) \cdot \alpha)-\int_{\mathscr{\mathscr { S }}}(K(\iota, t) \cdot \alpha)\right| \\
& \quad \leqq c_{0}\left[\int_{\mathscr{G}-\left(\mathscr{E}_{1} \cup \mathscr{E}_{2}\right)} \Theta(\iota, t, k)+\int_{\mathscr{E}_{1}} \Theta(\iota, t, k)+\int_{\mathscr{B}_{2}} \Theta(\iota, t, k)\right],
\end{aligned}
$$

where $\Theta(s, t, k)=|K(s, t+k)-K(s, t)|$. From the expression (11) for $K$, it follows by standard arguments that the first integral on the right is equal to or less than the product of $|A(t+k, t)|^{b}$ and some positive number.

For all $(s, t)$ such that $|A(s, t)| \leqq r$, let

$$
\begin{aligned}
X(s, t) & =\frac{\xi s-\xi t}{A(s, t)} & \text { if } A(s, t) \neq 0, & Y(s, t)=\frac{\eta s-\eta t}{A(s, t)} & \text { if } A(s, t) \neq 0 \\
& =D \xi s \quad \text { if } A(s, t)=0, & & =D \eta s & \text { if } A(s, t)=0 .
\end{aligned}
$$

Then, for each $s$ in $\mathscr{E}_{1} \cup \mathscr{E}_{2}, X(s, \iota), X(\iota, s), Y(s, \iota)$, and $Y(\iota, s)$ satisfy a Hölder condition on $\mathscr{E}_{1} \cup \mathscr{E}_{2}$ with exponent $b$ (see [4, p. 20]). Furthermore, $K=N / \pi A$, where

$$
N(s, t)=[Y(s, t) D \xi t-X(s, t) D \eta t] /\left[X^{2}(s, t)+Y^{2}(s, t)\right]
$$

Since, for some positive number $d_{0}$ and for all $(s, t)$ in $\mathscr{I} \times \mathscr{I}, X^{2}(s, t)+Y^{2}(s, t)>d_{0}$, it follows that $N(s, \iota)$ and $N(\iota, s)$ satisfy a Hölder condition on $\mathscr{E}_{1} \cup \mathscr{E}_{2}$ with exponent $b$, for each $s$ in $\mathscr{E}_{1} \cup \mathscr{E}_{2}$. 
Since $A(s, \iota)$ satisfies a Lipschitz condition for each $s$ in $\mathscr{E}_{1}$, it follows by Property (i) and Schwarz's inequality that

$$
\int_{\mathscr{\delta}_{1}} \Theta(\iota, t, k)=\frac{1}{\pi} \int_{\mathscr{E}_{1}}\left|\frac{N(\iota, t+k)}{A(\iota, t+k)}-\frac{N(\iota, t)}{A(\iota, t)}\right| \leqq c_{1}|A(t+k, t)|^{b} \log |A(t+k, t)|,
$$

for some positive number $c_{1}$.

By Property (i), for some positive numbers $c_{2}$ and $c_{3}$,

$$
\int_{\mathscr{E}_{2}} \Theta(\iota, t, k) \leqq \int_{\mathscr{E}_{2}}\left(\frac{c_{2}}{|A(\iota, t+k)|^{1-b}}+\frac{c_{2}}{|A(\iota, t)|^{1-b}}\right) \leqq c_{3}|A(t+k, t)|^{b},
$$

which completes the proof of Property (iv).

Property (v) can be proved as follows.

$$
\begin{aligned}
|H(s+h, t+k)-H(s, t)| \leqq & \int_{\mathscr{I}}(|K(s+h, \iota)+K(s, \iota)| \cdot|\Lambda(\iota, t+k)|) \\
& +\int_{\mathscr{T}}(|K(s, \iota)| \cdot|\Lambda(\iota, t+k)-\Lambda(\iota, t)|) \\
\leqq & \left(\int_{\mathscr{S}}|K(s+h, \iota)-K(s, \iota)|^{p_{1}}\right)^{1 / p_{1}}\left(\int_{\mathscr{I}}|\Lambda(\iota, t+k)|^{q_{1}}\right)^{1 / q_{1}} \\
& +\left(\int_{\mathscr{I}}|K(s, \iota)|^{p_{2}}\right)^{1 / p_{2}}\left(\int_{\mathscr{I}}|\Lambda(\iota, t+k)-\Lambda(\iota, t)|^{q_{2}}\right)^{1 / q_{2}}
\end{aligned}
$$

for properly chosen numbers $p_{1}, q_{1}, p_{2}, q_{2}$ such that $1 / p_{1}+1 / q_{1}=1,1 / p_{2}+1 / q_{2}=1$, $1 \leqq p_{1}$, and $1 \leqq p_{2}$.

The integral $\int_{s}|K(s+h, \iota)-K(s, \iota)|^{p_{1}}$ may now be treated like the integral $\int_{\mathscr{S}} \Theta(\iota, t, k)$ in the proof of (iv) above. The result is that, for some positive number $d_{1}$,

$$
\left(\int_{\mathscr{J}}|K(s+h, \iota)-K(s, \iota)|^{p_{1}}\right)^{1 / p_{1}} \leqq d_{1}|A(s+h, s)|^{\left(1 / p_{1}\right)-(1-b)}
$$

provided that $p_{1}(1-b)<1$.

Now $\Lambda(s, t)=B(s, t)+\log (1 /|A(s, t)|)$, where

$$
B(s, t)=\log \left(c / \sqrt{ }\left(X^{2}(s, t)+Y^{2}(s, t)\right)\right) .
$$

Since $B$ is continuous on $\mathscr{I} \times \mathscr{I}$ and $|\log (1 /|A(\iota, t)|)|^{q_{1}}$ is summable on $\mathscr{I}$ for every positive number $q_{1}$, it follows by the triangle inequality in $\mathscr{L}_{q_{1}}$ that

$$
\left(\int_{\sigma}|\Lambda(\iota, t+k)|^{q_{1}}\right)^{1 / q_{1}}
$$

exists and is finite.

Property (i) can be used to show that $\left(\int_{s}|K(s, \iota)|^{p_{2}}\right)^{1 / p_{2}}$ exists and is finite for every $p_{2}$ such that $p_{2}(1-b)<1$. 
The last integral in inequality (12) can be treated as follows:

$$
\begin{aligned}
\left(\int_{\mathscr{F}}|\Lambda(\iota, t+k)-\Lambda(\iota, t)|^{q_{2}}\right)^{1 / q_{2}} \leqq & \left(\int_{\mathscr{G}}|B(\iota, t+k)-B(\iota, t)|^{q_{2}}\right)^{1 / q_{2}} \\
& +\left(\int_{\mathscr{S}}|\log | \frac{A(\iota, t)}{A(\iota, t+k)}||^{q_{2}}\right)^{1 / q_{2}} .
\end{aligned}
$$

Since $X(s, \iota)$ and $Y(s, \iota)$ satisfy a Hölder condition with exponent $b$, so does $B(s, \iota)$, and hence

$$
\left(\int_{\mathscr{G}}|B(\iota, t+k)-B(\iota, t)|^{q_{2}}\right)^{1 / q_{2}} \leqq d_{2}|A(t+k, t)|^{b}
$$

for some positive number $d_{2}$. Now the second integral above may be written as a sum of integrals over $\mathscr{E}_{1}, \mathscr{E}_{2}$, and $\mathscr{I}-\left(\mathscr{E}_{1} \cup \mathscr{E}_{2}\right)$. Exploiting the Lipschitz condition satisfied by $A(s, \iota)$ in the first of these three integrals, applying Minkowski's inequality to the second, and making use of a well-known inequality for the logarithm function in the third gives that

$$
\left(\int_{\mathscr{S}}|\log | \frac{A(\iota, t)}{A(\iota, t+k)}||^{q_{2}}\right)^{1 / q_{2}} \leqq d_{3}\left(|A(t+k, t)|^{\left.1-b_{3}\right)^{1 / q_{2}}}\right.
$$

for some positive number $d_{3}$ and some $b_{3}$ such that $0<b_{3}<1$.

The proof that $\bar{H}$ satisfies a similar condition is almost exactly like that for $H$. This completes the proof of Property (v).

Property (vi) can be proved by induction. That it is true when $n=1$ can be seen from the expression obtained by differentiating the expression (11) for $K$. Since

$$
K_{n}(s, t)=\int_{\mathscr{\sigma}}\left(K(s, \iota) \cdot K_{n-1}(\iota, t)\right)=\int_{\mathscr{g}}\left(K_{n-1}(s, \iota) \cdot K(\iota, t)\right)
$$

and

$$
\int_{\mathscr{S}} K_{p}(s, \iota)=1
$$

for each positive integer $p$, it follows that

$$
\begin{aligned}
D_{1} K_{n}(s, t) & =\int_{\mathscr{S}}\left[D_{1} K(s, \iota) \cdot\left(K_{n-1}(\iota, t)-K_{n-1}(s, t)\right)\right] \\
& =\int_{\mathscr{S}}\left[D_{1} K_{n-1}(s, \iota) \cdot(K(\iota, t)-K(s, t))\right] .
\end{aligned}
$$

From the induction assumption and the symmetry of $D_{1} K$, it then follows by an integration by parts that

$$
\begin{aligned}
D_{1} K_{n}(t, s) & =\int_{\mathscr{f}}\left[D_{1} K(\iota, t) \cdot\left(K_{n-1}(\iota, s)-K_{n-1}(t, s)\right)\right] \\
& =(-1)^{n+1} \int_{\mathscr{\jmath}}\left[D_{1} K_{n-1}(s, \iota) \cdot(K(\iota, t)-K(s, t))\right]=(-1)^{n+1} D_{1} K_{n}(s, t) .
\end{aligned}
$$


The integration by parts is justified because the function

$$
(K(\iota, t)-K(s, t)) \cdot\left(K_{n-1}(\iota, s)-K_{n-1}(t, s)\right)
$$

is continuous on $\mathscr{B}$, and its derivative exists and is continuous except possibly at $s$ and $t$, and its derivative is summable on $\mathscr{I}$.

Property (vii) can now be proved as follows. By Properties (i) and (ii), for $p$ and $q$ sufficiently large, $D_{1} K_{p}(s, \iota)$ is summable and $K_{q}(\iota, t)$ is bounded, so that

$$
D_{1} K_{p+q}(s, t)=\int_{\mathscr{g}}\left(D_{1} K_{p}(s, \iota) \cdot K_{q}(\iota, t)\right),
$$

and hence $D_{1} K_{p+q}$ is bounded. Therefore, by Property (iv), $D_{1} K_{p+q+1}(s, \imath)$ satisfies a Hölder condition on $\mathscr{B}$ because

$$
D_{1} K_{p+q+1}(s, t)=\int_{\mathcal{S}}\left(D_{1} K_{p+q}(s, \iota) \cdot K(\iota, t)\right) .
$$

The fact that $D_{1} K_{p+q+1}(\iota, t)$ also satisfies a Hölder condition on $\mathscr{B}$ then follows from Property (vi).

\section{BIBLIOGRAPHY}

1. T. Carleman, Uber das Neumann-Poincarésche Problem für ein Gebiet mit Ecken, Uppsala Dissertation, 1917.

2. O. D. Kellogg, Potential functions on the boundary of their regions of definition, Trans. Amer. Math. Soc. 9 (1908), 39-50.

3. - Harmonic functions and Green's integral, Trans. Amer. Math. Soc. 13 (1912), 109-132.

4. N. I. Muskhelishvili, Singular integral equations, Noordhoff, Groningen, 1953.

5. W. J. Sternberg and T. L. Smith, The theory of potential and spherical harmonics, Univ. of Toronto Press, Toronto, 1946.

6. S. E. Warschawski, On the solution of the Lichtenstein-Gershgorin integral equation in conformal mapping, Nat. Bur. Standards Appl. Math. Ser. 42 (1955), 7-29.

7. A. C. Zaanen, Linear analysis, Noordhoff, Groningen, 1956.

ANDREWS UNIVERSITY,

Berrien Springs, Michigan 